\title{
SOVMM: Survey on Virtual Machine Migration
}

\author{
Koushalesh Acharya \\ M.Tech Scholar \\ Branch: CTA \\ TIEIT, Bhopal, M.P.
}

\author{
Amit Saxena \\ Head of Department \\ Dept: CSE \\ TIEIT, Bhopal, M.P.
}

\author{
Kaptan Singh \\ Professor \\ Dept: CSE \\ TIEIT, Bhopal, M.P.
}

\begin{abstract}
We are living in two worlds one where we physically present and one where we virtually present and that virtual world is nothing but an internet world. We spent lot of time on internet and our maximum work is on internet such as banking, business, and government work. Cloud Computing is the future of this world. Cloud computing is internet based computing technology. Virtual machine is the core element of this computing technology. In the data center many virtual machines present which satisfy the user request and their requirements. To efficiently satisfying the user need and uninterrupted services some time virtual machine needs to be migrated from one data center to another data center or from one host to another host within the data center. There are many algorithms for virtual machine migration which are based on different parameters and conditions. This paper comprises all such algorithms with their merits and demerits and also a comparative study of these algorithms.
\end{abstract}

\section{Keywords}

Cloud computing, virtual machine, virtual machine migration, hypervisor, virtual machine monitor, data center.

\section{INTRODUCTION}

Cloud computing is a model for enabling universal, suitable, on users demand network access to a sharable and configurable computing resources (e.g., networks, servers, storage, applications, and services) that can be rapidly provisioned and released minimum effort and minimum service provider's interaction[19]. From above definition it is clear that Cloud computing is on-demand pay-per-use technology where resources are shared. The thirst of sharing computing resources optimally among user's and organizations has leaded the gradual evolution of distributed, cluster and grid computing and finally now, we are in the age of Cloud Computing. It allows customers to provision resources on-demand, based on pay-as-you-go basis. The cloud data centers (CDCs) contain thousands of computing node connected through a high-speed network and consuming huge amount of electricity [2]. Mobile Internet services are typically provided by servers in cloud data center, usually with virtualization based cloud computing technologies. With the rapid growth of handheld devices and advancement of cloud computing technology, cloud data center has become widely known and effective way to provide resources for computing and storage. As a new computing model, cloud computing enables flexible management on the resources of computing, storage and networking. It also requires more advanced networking technologies like flexible control over the network traffic, network virtualization, dynamical network resource management, network programmability and so forth [3].

VM migration in cloud data center is very critical to both the user and the service provider at cloud data center. Virtualization is a key ingredient that has enabled such agility within a data center. For reconfiguring the computing resource in cloud data center, virtual machine (VM) migration plays a very important role in cloud computing system in terms of optimal resource scheduling and service migration. The servers provides services running on virtual machines could be move to a proper position, even if across geographical distances to satisfy the service requirements, the defined policies of the cloud data center or green computing purpose and so on without interruption.

With the rapid growth of cloud computing, virtualization has become a popular practice in the design of data centers. To achieve the better utilization, failure tolerance, energy efficiency and load balance service providers can migrate the VMs without considering the running status of the user applications [7]. Some strong recent efforts have been made to lower the cost of migrating one or several VMs from an initial placement to another given destination placement, namely the migration phase.

What is live Virtual machine migration: Clark et al. [18] first present a systematic live migration mechanism with practically small service downtime for a single VM migration. Live migration of virtual machine is the migration of virtual machine in a running environment of cloud computing. While migration none of the services of user is interrupted and stopped. User does not even realize any kind of service break down in its utilization of resources. Efficient live migration of virtual machines is very much important for SLA (service level agreement). Each migration decision depends on three questions: when to move a virtual machine, which virtual machine to move and where to move it? In this paper we summarize different live migration techniques of virtual machines with their merits and demerits and also a comparative study of all these algorithms.

The rest of the paper is organized is as follows: section 2 discusses need of virtual machine migration. section 3 discuss related works, section 4 discuss the comparison of these algorithms and last section is conclusion.

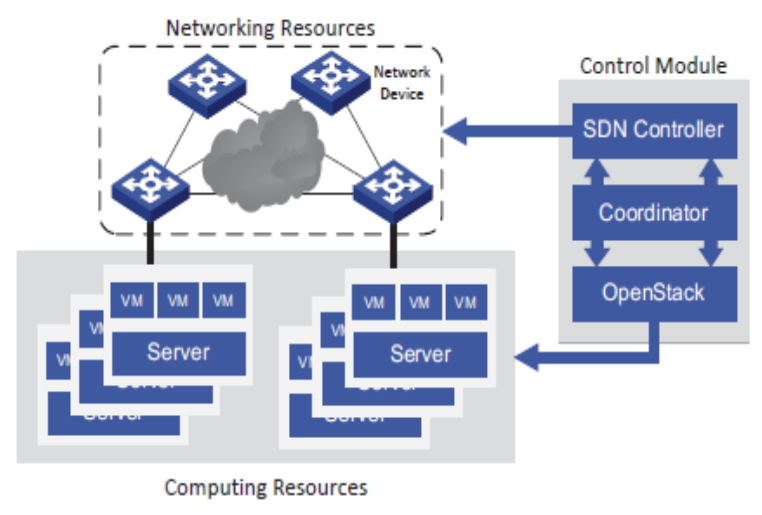

Fig 1: Model of Cloud computing 


\section{NEED OF VIRTUAL MACHINE MIGRATION}

Workload on servers keeps changing dynamically. It's not possible to provision additional servers manually. So, VMM accommodates for the changing workloads automatically (It can adapt to both provisioned and unprovisioned workloads) [20].

Normally scheduled maintenance results in some downtime for the users. But with Virtual Machine Migration, the VM can be migrated to some other server (for that period of scheduled maintenance) and brought back to the host server after the maintenance is completed. Virtual Machine Migration can also migrate Virtual Machines in case of unscheduled server downtime (due to some fault in server), so that users experience high availability of applications at all times.

Virtual Machine Migration can also be applying for Disaster Recovery. This process involves setting up of similar resources in a disaster recovery site with high speed WAN links and specialized network connectivity equipment. The Virtual machines states are frequently synchronized (replicated) between the primary servers and the servers in the disaster recovery site, so that migration can happen quickly in case of a disaster[20]

It's very hard to migrate operating system and application(s) from one server to another server and easy to migrate a Virtual Machine from one server to another server. Using Virtual Machine Migration, it's possible to migrate operating system(s) and application(s) without disrupting the services from older servers to newer servers easily..

\section{RELATED WORKS}

Using of hardware and software resources to create the perception that one or more entities exist, although the entities do not physically exist is called the virtualization. Virtualization, allow many operating systems to run on single physical environment [4].

Many modern hypervisors which is also called Virtual Machine Monitors or VMM do support live VM migration. A key objective during migration is to lower the downtime of the service provided by the VM. The total migration time is the difference between when a migration is initiated until the time when the original VM may be discarded. Different mechanisms can be used for live VM migration. S. Achleitner et. al. proposed a method of Stealth Migration viz hiding virtual machine on the network while migration. For the security reasons this is very much important for the security of virtual machine during migration.

Huandong Wang et. al. presented a technique that how to decrease the total migration time in software defined network (SDN) scenarios. Why SDN? Because SDN has a centralized controller, it is very easy to get the global view of the network, such as the topology, bandwidth utilization on each path, and other performance parameters. On the other hand, SDN provides a flexible way to set forwarding rules so that we can provide multipath forwarding between the migration source and destination. In SDN, the forwarding rules or we can say the routing methods are installed dynamically and we can devide the traffic on any path arbitrarily. We allow multiple VMs can be migrated parallely via multiple routing paths. The objective of Huandong Wang is to optimize the total migration time by determining their migration orders and transmission rates.

Ryo Watanabe et. al. described algorithm for energy-aware virtual machine migration policy in a scalable cluster of servers. In his research paper he mentioned VM selection policy for migration. That vm selection policy is that select a hosting sever for the process whose expected electric energy consumption is minimum. Then select a vm on the hosted server where are number of resident processes is minimum. Then after perform the process on the selected virtual machine of the hosted server. According to results his algorithm called MSVM (modified simple virtual machine migration). In their research they showed that electric energy consumption and active time of servers can be reduced to $80 \%$. Megh a dynamic virtual machine migration algorithm on the basis of learn-as-you-go basis[8].

Which means that megh has the capacity to learn uncertain dynamics in real time? Fig 2 shows Megh reaches the almost stable cost per step in around 100 steps because of the initial learning phase. Being a greedy heuristics, T H R-M M T faces high variance and instability even after the initial convergence. Fig(B) shows Mad VM and Megh, being reinforcement learning algorithms, have similar trends of convergence. But Megh reduces the expenditure by $14.25 \%$ and $4.3 \%$ with respect to that of T H R-M M T and Mad VM respectively. It validates the cost effectiveness, robustness and stability of Megh for optimal resource management for a diverse set of workloads with respect to other heuristics. Ahmed Sallam et. al. uses a multi-objective virtual machine migration policy for virtual machine migration in cloud environment.

Fig 2: Comparative performance analysis (per-step cost (in SGD))

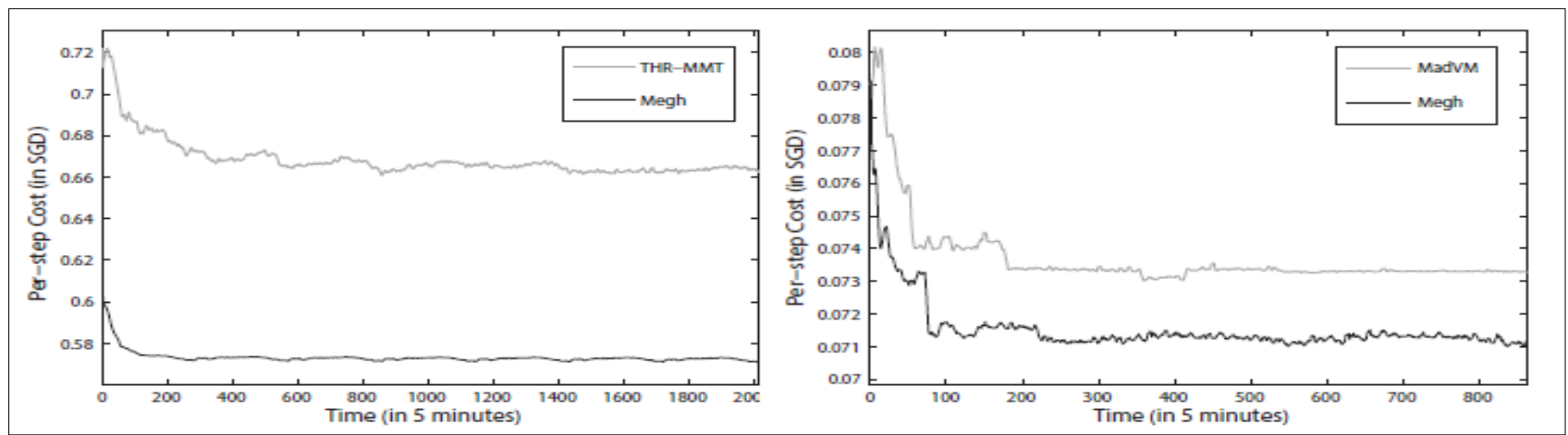


Table 1: Comparative study of VM migration techniques

\begin{tabular}{|c|c|c|c|c|c|}
\hline \multicolumn{3}{|c|}{ Virtual machine migration techniques } & Concept & Pros & Cons \\
\hline \multicolumn{3}{|c|}{ Stop-and-Copy Approach[10] } & $\begin{array}{l}\text { Halting the target VM, Copying } \\
\text { all pages to the destination and } \\
\text { then start execution of that VM }\end{array}$ & Simplicity & $\begin{array}{c}\text { More Service } \\
\text { downtime }\end{array}$ \\
\hline \multicolumn{3}{|c|}{ Pre-copy approach[11] } & $\begin{array}{l}\text { Transfer VM's memory contents } \\
\text { first then processor states are } \\
\text { transferred to destination. }\end{array}$ & $\begin{array}{l}\text { 1. Application downtime } \\
\text { caused by migration is less } \\
\text { than a second. } \\
\text { 2. Migration is completely } \\
\text { transparent to the } \\
\text { application, to the operating } \\
\text { system. }\end{array}$ & $\begin{array}{l}\text { Duplicate } \\
\text { transmission } \\
\text { overhead. }\end{array}$ \\
\hline \multicolumn{3}{|c|}{ Adaptive rate limiting approach[12] } & $\begin{array}{l}\text { Used the concept of writable } \\
\text { working set and showed OS } \\
\text { migration build on top of the } \\
\text { VMM. }\end{array}$ & $\begin{array}{l}\text { Service downtime as low as } \\
60 \mathrm{~ms} \text {. }\end{array}$ & $\begin{array}{l}\text { Large total } \\
\text { migration. }\end{array}$ \\
\hline \multirow{4}{*}{$\begin{array}{l}\text { Improved } \\
\text { Pre-copy } \\
\text { approach }\end{array}$} & \multirow{2}{*}{$\begin{array}{l}\text { Memory } \\
\text { compression } \\
\text { technique }\end{array}$} & $\begin{array}{c}\text { Adaptive } \\
\text { memory } \\
\text { compression[13] }\end{array}$ & $\begin{array}{l}\text { Memory compression based VM } \\
\text { migration approach that first uses } \\
\text { memory compression. }\end{array}$ & $\begin{array}{l}\text { 1. It provides fast and stable } \\
\text { virtual machine migration. } \\
\text { 2. Minimize the downtime } \\
\text { and total migration time. }\end{array}$ & $\begin{array}{l}\text { Compression } \\
\text { operations } \\
\text { introduced } \\
\text { additional } \\
\text { overhead. }\end{array}$ \\
\hline & & $\begin{array}{c}\text { Delta } \\
\text { compression[14] }\end{array}$ & $\begin{array}{l}\text { The dalta compression live } \\
\text { migration algorithm is } \\
\text { implemented as a notification to } \\
\text { the KVM hypervisor. }\end{array}$ & $\begin{array}{l}\text { 1. Downtime is reduced. } \\
\text { 2. Increased migration } \\
\text { throughput. }\end{array}$ & $\begin{array}{l}\text { Compression } \\
\text { operations } \\
\text { introduced } \\
\text { additional } \\
\text { overhead. }\end{array}$ \\
\hline & \multirow[t]{2}{*}{$\begin{array}{l}\text { Frequently } \\
\text { updated pages of } \\
\text { memory. }\end{array}$} & $\begin{array}{l}\text { Time series } \\
\text { prediction } \\
\text { technique[15] }\end{array}$ & $\begin{array}{l}\text { With time series prediction } \\
\text { technique, it identified frequently } \\
\text { updated dirty pages in the past } \\
\text { and future period more precisely } \\
\text { and transmits them on the last } \\
\text { round of iteration. }\end{array}$ & $\begin{array}{l}\text { 1. Less number of iteration. } \\
\text { 2. Less downtime and } \\
\text { migration time. }\end{array}$ & $\begin{array}{l}\text { This approach } \\
\text { is useful when } \\
\text { high dirty } \\
\text { pages in } \\
\text { iteration. }\end{array}$ \\
\hline & & $\begin{array}{l}\text { Detecting } \\
\text { memory update } \\
\text { patterns[16] }\end{array}$ & $\begin{array}{l}\text { Detecting memory update } \\
\text { patterns and terminating } \\
\text { migration. }\end{array}$ & $\begin{array}{l}\text { Provide minimal downtime } \\
\text { and minimal impact on end } \\
\text { to end application } \\
\text { performance. }\end{array}$ & $\begin{array}{l}\text { Useful for } \\
\text { memory } \\
\text { intensive } \\
\text { applications. }\end{array}$ \\
\hline
\end{tabular}

\section{CONCLUSION}

This paper presents a review of various live virtual machine migration techniques in cloud computing. Few techniques proposed by researchers other than these two approaches are also discussed. The paper also discussed VM migration techniques for cloud federation. All the techniques discussed above try to minimize the total downtime of migration and provide better performance in low bandwidth. Then the various scenarios are presented where the migration of virtual machine are needed. The comparative study has been made on different live virtual machine migration techniques with their pros and cons.

\section{REFERENCES}

[1] S. Achleitner, T. La Porta and P. McDaniel, "Stealth Migration: Hiding Virtual Machines on the Network" "IEEE INFOCOM 2017 - IEEE Conference on Computer Communications", 2017.

[2] Anita Choudhary, M. C. Govil, Girdhari Singh, Lalit K. Awasthi, E.S. Pilli,Nitin Kumar, "Improved Virtual Machine migration approaches in Cloud Environment", "IEEE International Conference on Cloud Computing in Emerging Markets”, 2016.

[3] Bo Hu, Member, IEEE, Shanzhi Chen, Senior Member, IEEE, Jianye Chen, Zhangfeng $\mathrm{Hu}$, "A MobilityOriented Scheme for Virtual Machine Migration in 
Cloud Data Center Network ", "National Natural Science Foundation of China for Distinguished Young Scholar under Grant 61425012 ”.

[4] Gupta P., Vishwakarma P., Patel A., "Power - Aware Virtual Machine Consolidation considering Multiple Resources with Live Migration", "International Journal of Computer Applications (0975 - 8887) Volume 103 No 17, October 2014"

[5] Huandong Wang, Yong Li, Ying Zhang, Depeng Jin, "Virtual Machine Migration Planning in SoftwareDefined Networks", "IEEE transaction on Cloud Computing", 2016.

[6] Ryo Watanabe, Tomoya Enokido, "Energy-aware Virtual Machine Migration Models in a Scalable Cluster of Servers", "IEEE 31st International Conference on Advanced Information Networking and Applications", (85-92), 2017.

[7] R. Lee, B. Jeng, "Load-Balancing Tactics in Cloud", IEEE International Conference on Cyber-Enabled Distributed Computing and Knowledge Discovery, pp. 447-454, 2011.

[8] Debabrota Basu, Xiayang Wang, Yang Hong, Haibo Chen, Stéphane Bressan, "Learn-as-you-go with Megh: Efficient Live Migration of Virtual Machines", "IEEE 37th International Conference on Distributed Computing Systems", 2017.

[9] Ahmed Sallam, and Kenli Li, “ A Multi-objective Virtual Machine Migration Policy in Cloud Systems", "The Computer Journal Advance", 2013.

[10] C. P. Sapuntzakis, R. Chandra, B. Pfaff, J. Chow, M. S. Lam, and M. Rosenblum 2002. Optimizing the migration of virtual computers. Proceedings of the 5th Symposium on Operating Systems Design and Implementation (OSDI-02) ACM digital Library.

[11] M. Nelson, B. Lim, and G. Hutchines 2005. Fast transparent migration for virtual machines. Proceedings of the USENIX Annual Technical Conference (USENIX'05), 391-394.

[12] C. Christopher, F. Keir, H. Steven, H. Jacob Gorm, J. Eric, L. Christian, P. Ian, and W. Andrew 2005. Live migration of virtual machines. 2nd conference on Symposium on Networked Systems Design \& Implementation - Volume 2: USENIX Association.

[13] C. Christopher, F. Keir, H. Steven, H. Jacob Gorm, J. Eric, L. Christian, P. Ian, and W. Andrew 2005. Live migration of virtual machines. 2nd conference on Symposium on Networked Systems Design \& Implementation - Volume 2: USENIX Association.

[14] P. Svard, J. Tordsson, B. Hudzia, and E. Elmroth 2011. High performance live migration through dynamic page transfer reordering and compression. 3rd IEEE International Conference on Cloud Computing Technology and Science, CloudCom, 542-548.

[15] F. Ma, F. Liu, and Z. Liu 2010. Live Virtual Machine Migration Based on Improved Precopy Approach. Proceedings Software Engineering and Service Sciences, 230-233.

[16] B.Hu, Z. Lei, Y. Lei, D. Xu and J. Li 2011. A TimeSeries Based Precopy Approach for Live Migration of Virtual Machine. IEEE 17th International Conference on Parallel and Distributed Systems, 947-952.

[17] K. Z. Ibrahim, S. Hofmeyr, C. Iancu, and E. Roman 2011. Optimized precopy live migration for memory intensive applications. International Conference for High Performance Computing, Networking, Storage and Analysis (SC), 1-11.

[18] C. Clark, K. Fraser, S. Hand, J. G. Hansen, E. Jul, C. Limpach, I. Pratt, and A. Warfield, "Live migration of virtual machines," in NSDI 2005.

[19] NIST White paper.

[20] Rajesh K article of "Importance of virtual machine migration in server virtualization”, May 2011. 